University of Wollongong

Research Online

Faculty of Engineering - Papers (Archive)

Faculty of Engineering and Information

Sciences

8-8-2001

\title{
Terahertz-photon-modified magnetotransport in a semiconductor in Voigt geometry
}

W. Xu

University of Wollongong

I. Khmyrova

University of Aizu, Japan

V. Ryzhii

University of Aizu, Japan

Follow this and additional works at: https://ro.uow.edu.au/engpapers

Part of the Engineering Commons

https://ro.uow.edu.au/engpapers/241

\section{Recommended Citation}

Xu, W.; Khmyrova, l.; and Ryzhii, V.: Terahertz-photon-modified magnetotransport in a semiconductor in Voigt geometry 2001.

https://ro.uow.edu.au/engpapers/241

Research Online is the open access institutional repository for the University of Wollongong. For further information contact the UOW Library: research-pubs@uow.edu.au 


\title{
Terahertz-photon-modified magnetotransport in a semiconductor in Voigt geometry
}

\author{
W. $\mathrm{Xu}^{*}$ \\ Department of Engineering Physics, University of Wollongong, Wollongong, New South Wales 2522, Australia \\ I. Khmyrova and V. Ryzhii \\ Computer Solid State Physics Laboratory, University of Aizu, Aizu-Wakamatsu 965-8580, Japan
}

(Received 23 January 2001; published 8 August 2001)

\begin{abstract}
We present a theoretical study of transport and optical properties of a semiconductor-based electron gas subjected simultaneously to quantizing magnetic fields and intense terahertz (THz) laser fields in Voigt geometry. It is found that the presence of the $\mathrm{THz}$ radiation can result in an enhanced magnetophonon resonance effect and a resonant-absorption peak can be observed at about $f \sim 1 \mathrm{THz}$ for GaAs in high magnetic fields. The results are pertinent to experiments where $\mathrm{THz}$ free-electron lasers are employed as intense radiation sources.
\end{abstract}

DOI: 10.1103/PhysRevB.64.085209

PACS number(s): 72.10.Di, 72.20.My, 78.20.Bh

\section{INTRODUCTION}

When we consider a semiconductor-based electron gas subjected simultaneously to quantizing magnetic fields and intense terahertz ${ }^{1}(\mathrm{THz})$ laser fields, we enter a regime with different competing energies where the electron kinetic energy, cyclotron energy, phonon energy, photon energy, etc., are of the order of meV. Hence, by applying intense $\mathrm{THz}$ fields to a semiconductor in quantizing magnetic fields, we can investigate high-field magneto-optical properties and photon-modified magnetotransport in an electron gas system. At present, intense $\mathrm{THz}$ radiation can be provided by $\mathrm{THz}$ free-electron lasers (FEL's), which are coherent, high-power (the output power can reach up to $100 \mathrm{~kW} / \mathrm{cm}^{2}$ ), frequencytunable $(f \sim 0.1-10 \mathrm{THz})$, and linearly polarized radiation sources. $^{2}$

In semiconductor materials such as $\mathrm{GaAs}$ and $\mathrm{Si}$, the energy gaps $\left(E_{g} \sim \mathrm{eV}\right)$ among different bands and valleys are much larger than the energy of $\mathrm{THz}$ photons $(\hbar \omega \sim \mathrm{meV})$. Thus, under $\mathrm{THz}$ radiation the electronic scattering via interband and intervalley transitions in these materials can be neglected when the radiation intensity is not extremely high. This implies that the electron-photon-phonon interaction is the limiting factor in determining transport and optical properties of these semiconductor materials in the presence of intense $\mathrm{THz}$ radiation. When a linearly polarized radiation field and a static magnetic field are applied simultaneously to an electron gas system, there are two different configurations that can be used to study the response of electrons to these fields. In the Voigt geometry, the linearly polarized radiation field does not couple to the magnetic field, whereas in the Faraday geometry the radiation field couples to the magnetic field. In the presence of an intense $\mathrm{THz}$ laser field and a quantizing magnetic field, some interesting magneto-optical properties of semiconductors have been investigated in recent theoretical work, such as magnetophoton-phonon resonances ${ }^{3}$ and the dynamical and magneto-optical FranzKeldysh effect. ${ }^{4,5}$

Recently, experimental work has been conducted to investigate magneto-optical and magnetotransport properties in GaAs-based systems in quantizing magnetic fields and $\mathrm{THz}$ FEL fields. ${ }^{6}$ The prime motivation of this work was to exam- ine THz-photon-modified high-field and high-temperature magnetotransport phenomena such as the magnetophonon resonance (MPR) effect. However, these measurements were carried out in the Faraday geometry (i.e., a magnetic field is applied along the $z$ direction and a laser field is applied parallel to the magnetic field and is polarized linearly along the $x$ direction) for GaAs-based two-dimensional systems, where the intense $\mathrm{THz}$ laser field couples strongly to the static magnetic field. As a consequence, only a pronounced cyclotron resonance (CR) effect ${ }^{7}$ could be observed and the MPR effect could not survive in the measurement of resistivity $\rho_{x x}$.

In order to examine a THz-photon-modified quantum resonance effect such as MPR in magnetotransport measurements, it is necessary for us to suppress the CR effect. One option is to undertake the measurements in the Voigt geometry where the laser field does not couple directly to the magnetic field and, therefore, the CR effect is no longer present. The motivation of this paper is to study theoretically how electrons in a semiconductor interact with linearly polarized intense $\mathrm{THz}$ laser fields in the presence of quantizing magnetic fields in the Voigt geometry. In Sec. II, we study the magnetophoton-phonon interactions in this geometry. From these results, in Sec. III we derive the momentum- and energy-balance equations based on the Boltzmann equation. These balance equations can be used to calculate magnetotransport and magneto-optical coefficients for an electron gas system. The results obtained from this study are discussed in Sec. IV and summarized in Sec. V.

\section{MAGNETOPHOTON-PHONON INTERACTIONS}

In this paper, we consider the situation where a magnetic field $B$ is applied along the $z$ direction of an ideal threedimensional electron gas (3DEG), and a laser field $A(t)$ is applied along the $x$ direction and is linearly polarized along the $z$ direction. In this configuration (known as the Voigt geometry), the time-dependent single-electron Schrödinger equation can be solved analytically and the effect of the radiation and magnetic fields can be included exactly within the time-dependent electron wave function $\psi_{N, k_{y}, k_{z}}(\mathbf{R}, t) .{ }^{4} \mathrm{It}$ has been demonstrated ${ }^{8}$ that for an electron-photon-phonon system (i) the unitary operator in the presence of the electro- 
magnetic (EM) field, $U_{0}\left(t, t^{\prime}\right)$, can be determined from the time-dependent electron wave function when electronphonon scattering is absent; (ii) with $U_{0}\left(t, t^{\prime}\right)$, the unitary operator in the presence of the electron-phonon scattering, $U\left(t, t^{\prime}\right)$, can be derived using time-dependent perturbation theory, where only the electron-phonon interaction is treated as a perturbation; and (iii) from $U\left(t, t^{\prime}\right)$ the steady-state (i.e., $\left.t-t^{\prime} \rightarrow \infty\right)$ electronic transition rate can be obtained in the presence of the radiation field and of the electron-phonon interaction. Applying these theoretical approaches to the case of a 3DEG subjected to EM and magnetic fields in the Voigt geometry, the first-order steady-state transition probability induced by magnetophoton-phonon scattering is obtained as

$$
\begin{aligned}
W_{N^{\prime} N}\left(k_{z}^{\prime}, k_{z}\right)= & \frac{2 \pi}{\hbar} \sum_{\mathbf{q}}\left[N_{Q}+\frac{1}{2} \mp \frac{1}{2}\right]\left|V_{\mathbf{Q}}\right|^{2} \\
& \times C_{N^{\prime} N}\left(l^{2} q^{2} / 2\right) \delta_{k_{y}^{\prime}, k_{z}+q_{z}} \\
& \times \sum_{m=-\infty}^{\infty} J_{m}^{2}\left(r_{0} q_{z}\right) \delta\left[E_{N^{\prime}}\left(k_{z}^{\prime}\right)\right. \\
& \left.-E_{N}\left(k_{z}\right)-m \hbar \omega \mp \hbar \omega_{Q}\right] .
\end{aligned}
$$

Here, $N$ is the Landau-level (LL) index, $k_{z}$ is the electron wave vector along the $z$ direction, $\mathbf{Q}=\left(\mathbf{q}, q_{z}\right)=\left(q_{x}, q_{y}, q_{z}\right)$ is the phonon wave vector, $\hbar \omega_{Q}$ is the phonon energy, $N_{Q}$ $=\left(e^{\hbar \omega_{Q} / k_{B} T}-1\right)^{-1}$ is the phonon occupation number, $V_{\mathbf{Q}}$ is the electron-phonon interaction coefficient, $E_{N}\left(k_{z}\right)$ $=E_{N}+\hbar^{2} k_{z}^{2} / 2 m^{*}, m^{*}$ is the effective electron mass, $E_{N}=(N+1 / 2) \hbar \omega_{c}$ is the $N$ th LL energy with $\omega_{c}=e B / m^{*}$ being the cyclotron frequency, $C_{N, N+J}(x)=[N ! /(N$ $+J) !] x^{J} e^{-x}\left[L_{N}^{J}(x)\right]^{2}$ with $L_{N}^{J}(x)$ being the associated Laguerre polynomials, $l=(\hbar / e B)^{1 / 2}$, and the upper (lower) case refers to absorption (emission) of a phonon. Furthermore, in Eq. (1), $m$ is an index for $m$-photon absorption $(m$ $>0)$ or emission $(m<0)$ and $m=0$ for elastic optic scattering, $r_{0}=e F_{0} /\left(m^{*} \omega^{2}\right)$ with the dimension of length, $\omega$ and $F_{0}$ are, respectively, the frequency and the electric field strength of the radiation field, and $J_{m}(x)$ is a Bessel function.

Equation (1) exhibits features distinctive for magnetophoton-phonon interactions in an electron gas system in the Voigt geometry. When an intense EM field is present, electrons in the system can interact with the radiation field via channels for optical emission and absorption. In the Voigt geometry, the emission and absorption of photons by electrons can be achieved via indirect optical channels mediated by electron-phonon scattering. On the other hand, the emission and absorption of phonons via electronic transition events are accompanied by the emission and absorption of photons. For the case of a high-frequency $(\omega \gg 1)$ and/or low-intensity $\left(F_{0} \ll 1\right)$ radiation, entailing $r_{0} \rightarrow 0$, Eq. (1) becomes that obtained by using Fermi's golden rule when the radiation field is absent. From Eq. (1) we see that the change of electron wave vector or momentum along the direction where the radiation field is polarized, $q_{z}$, plays an essential role in switching different optical channels for magnetophoton-phonon scattering, through the term $J_{M}^{2}\left(r_{0} q_{z}\right)$ in Eq. (1).
For polar semiconductors such as GaAs, the frequency of the phonon oscillation associated with longitudinal-optic (LO) modes is at about $\mathrm{THz}$ frequency $\left(\omega_{\mathrm{LO}} / 2 \pi\right.$ $\simeq 8.85 \mathrm{THz}$ ). This suggestes that the electron-LO-phonon coupling is the principal channel for relaxation of $\mathrm{THz}-$ excited electrons in GaAs, due to its large energy transfer during a scattering event. For electron-LO-phonon scattering, (i) $\omega_{Q} \simeq \omega_{\mathrm{LO}}$, the LO-phonon frequency in the longwavelength range; (ii) $N_{Q} \simeq N_{0}=\left(e^{\hbar \omega_{\mathrm{LO}} / k_{B} T}-1\right)^{-1}$; and (iii) the coupling coefficient is given by the Frölich Hamiltonian $\left|V_{\mathbf{Q}}\right|^{2}=4 \pi \alpha L_{0}\left(\hbar \omega_{\mathrm{LO}}\right)^{2} / Q^{2}$, where $\alpha$ is the electron-LOphonon coupling constant and $L_{0}=\left(\hbar / 2 m^{*} \omega_{\mathrm{LO}}\right)^{1 / 2}$ is the polar radius. Thus, the transition rate induced by magnetophoton-LO-phonon interaction reads

$$
\begin{aligned}
W_{N^{\prime} N}\left(k_{z}^{\prime}, k_{z}\right)= & 8 \pi^{2} \alpha L_{0} \hbar \omega_{\mathrm{LO}}^{2}\left[N_{0}+\frac{1}{2} \mp \frac{1}{2}\right] \\
& \times \sum_{\mathbf{q}} \frac{C_{N^{\prime} N}\left(l^{2} q^{2} / 2\right)}{q^{2}+q_{z}^{2}} \delta_{k_{z}^{\prime}, k_{z}+q_{z}} \\
& \times \sum_{m=-\infty}^{\infty} J_{m}^{2}\left(r_{0} q_{z}\right) \delta\left[E_{N^{\prime}}\left(k_{z}^{\prime}\right)\right. \\
& \left.-E_{N}\left(k_{z}\right)-m \hbar \omega \mp \hbar \omega_{\mathrm{LO}}\right] .
\end{aligned}
$$

\section{MOMENTUM- AND ENERGY-BALANCE EQUATIONS}

In the present study, we consider a situation where the stationary transport and optical properties are investigated by conventional transport measurements. That is, an external dc probing field $E_{z}$ (or current $I_{z}$ ) is applied along the $z$ direction, the current (or voltage) is measured along this direction, and the longitudinal resistivity $\rho_{z z}$ and the electron-energyloss rate (EELR) are deduced from these data. In this case, a semiclassical transport equation such as the Boltzmann equation can be used as a governing transport equation to calculate quantities such as $\rho_{z z}$ and the EELR. The steady-state Boltzmann equation in the case of nondegenerate statistics can be written as

$$
\begin{aligned}
\frac{F}{\hbar} \frac{\partial f_{N}\left(k_{z}\right)}{\partial k_{z}}= & g_{s} \sum_{N^{\prime}, k_{z}^{\prime}}\left[f_{N^{\prime}}\left(k_{z}^{\prime}\right) W_{N N^{\prime}}\left(k_{z}, k_{z}^{\prime}\right)\right. \\
& \left.-f_{N}(k) W_{N^{\prime} N}\left(k_{z}^{\prime}, k_{z}\right)\right],
\end{aligned}
$$

where $f_{N}\left(k_{z}\right)$ is the steady-state momentum-distribution function for an electron in the state $\left|N, k_{z}\right\rangle, g_{s}=2$ accounts for spin degeneracy, $W_{N^{\prime} N}\left(k_{z}^{\prime}, k_{z}\right)$ is the steady-state electronic transition rate for an electron scattered from state $\left|N, k_{z}\right\rangle$ to state $\left|N^{\prime}, k_{z}^{\prime}\right\rangle$, and $F=-e E_{z}$ is the external force acting on the electron. It should be noted that in the present problem the effect of the radiation field has already been included within the electronic transition rate and, hence, the radiation field no longer appears in the force term of the Boltzmann equation.

Using the balance equation approach based on the Boltzmann equation, the idea is to choose a certain form for the electron distribution function. After assuming that the electron momentum-distribution function can be described by a 
statistical energy distribution function via $f_{N}\left(k_{z}\right) \simeq f(\epsilon)$, where $\epsilon=E_{N}+\hbar^{2}\left(k_{z}-m^{*} v_{z} / \hbar\right)^{2} / 2 m *$ drifted by the average electron velocity $v_{z}$ along the $z$ direction due to the presence of the electric field $E_{z}$, for the first and second moments the momentum- and energy-balance equations can be derived by multiplying, respectively, $\Sigma_{N, k_{z}} k_{z}$ and $\Sigma_{N, k_{z}} E_{N}\left(k_{z}\right)$ on both sides of the Boltzmann equation. Thus, two equations can be obtained:

$$
e E_{z}=\frac{2 \hbar}{\pi N_{e} l^{2}} \sum_{N^{\prime}, N} \sum_{k_{z}^{\prime}, k_{z}}\left(k_{z}^{\prime}-k_{z}\right) f(\epsilon) W_{N^{\prime} N}\left(k_{z}^{\prime}, k_{z}\right)
$$

and

$$
\begin{aligned}
\frac{1}{2} e E_{z} N_{e} v_{z}= & \frac{1}{\pi l^{2}} \sum_{N^{\prime}, N} \sum_{k_{z}^{\prime}, k_{z}}\left[E_{N^{\prime}}\left(k_{z}^{\prime}\right)-E_{N}\left(k_{z}\right)\right] \\
& \times f(\epsilon) W_{N^{\prime} N}\left(k_{z}^{\prime}, k_{z}\right) .
\end{aligned}
$$

Here $N_{e}$ is the total electron density in the system and the condition of electron-number conservation has been used where $N_{e}=\left(\pi^{2} l^{2}\right)^{-1} \Sigma_{N} \int_{0}^{\infty} d k_{z} f\left(E_{N}\left(k_{z}\right)\right)$. For the case of a weak probing field $E_{z}$, the average electron velocity $v_{z}$ is also small. Expanding $f(\epsilon)$ with respect to $v_{z}$ gives

$$
f(\epsilon) \simeq f\left(E_{N}+E\right)-\hbar v_{z} k_{z} \frac{\partial f\left(E_{N}+E\right)}{\partial E},
$$

where $E=\hbar^{2} k_{z}^{2} / 2 m^{*}$. After using this approximation and noting that the longitudinal resistivity is defined by

$$
\rho_{z z}=\frac{1}{N_{e} e \mu_{z z}}=\frac{m^{*}}{N_{e} e^{2} \tau}=-\frac{E_{z}}{N_{e} e v_{z}},
$$

the momentum-relaxation time $\tau$ can be calculated from the momentum-balance equation through

$$
\frac{1}{\tau}=\frac{2 \hbar \omega_{c}}{\pi N_{e}} \sum_{N^{\prime}, N} \sum_{k_{z}^{\prime}, k_{z}} k_{z}\left(k_{z}^{\prime}-k_{z}\right) W_{N^{\prime} N}\left(k_{z}^{\prime}, k_{z}\right) \frac{\partial f\left(E_{N}+E\right)}{\partial E} .
$$

Furthermore, the electron-energy-loss rate per electron is defined as $P=\sigma_{z z} E_{z}^{2} / 2 N_{e}$ with $\sigma_{z z}=1 / \rho_{z z}$, which can be determined from the energy-balance equation through

$$
\begin{aligned}
P= & -\frac{1}{\pi l^{2}} \sum_{N^{\prime}, N} \sum_{k_{z}^{\prime}, k_{z}}\left[E_{N^{\prime}}\left(k_{z}^{\prime}\right)-E_{N}\left(k_{z}\right)\right] W_{N^{\prime} N}\left(k_{z}^{\prime}, k\right) \\
& \times f\left(E_{N}+E\right) .
\end{aligned}
$$

Introducing the transition rate induced by magnetophoton-LO-phonon scattering, Eq. (2), into the momentumand energy-balance equations, Eqs. (7) and (8), we have

$$
\begin{aligned}
\frac{1}{\tau}= & -\frac{2 \alpha L_{0} \omega_{\mathrm{LO}^{m}}^{2} *}{\pi^{2} \hbar N_{e} l^{2}}\left[N_{0}+\frac{1}{2} \mp \frac{1}{2}\right] \\
& \times \sum_{m=-\infty}^{\infty} \sum_{N^{\prime}, N} \int_{0}^{\infty} \frac{d q_{z}}{q_{z}} X_{N^{\prime} N}\left(l^{2} q_{z}^{2} / 2\right) J_{m}^{2}\left(r_{0} q_{z}\right) \\
& \times\left.\left(E_{q_{z}}+\epsilon_{N^{\prime} N m}^{\mp}\right) \frac{\partial f\left(E_{N}+E\right)}{\partial E}\right|_{E=\left(E_{q_{z}}+\epsilon_{N^{\prime} N m}^{\mp}\right)^{2} / 4 E_{q_{z}}}
\end{aligned}
$$

and

$$
P=\sum_{m=-\infty}^{\infty}\left(m \hbar \omega \pm \hbar \omega_{\mathrm{LO}}\right) \lambda_{m}^{\mp}
$$

with the inverse of the energy-relaxation time for the $m$ th optical process

$$
\begin{aligned}
\lambda_{m}^{\mp}= & \frac{\alpha L_{0} \omega_{\mathrm{LO}}^{2} m^{*}}{\pi^{2} \hbar N_{e} l^{2}}\left[N_{0}+\frac{1}{2} \mp \frac{1}{2}\right] \\
& \times \sum_{N^{\prime}, N} \int_{0}^{\infty} \frac{d q_{z}}{q_{z}} X_{N^{\prime} N}\left(l^{2} q_{z}^{2} / 2\right) J_{m}^{2}\left(r_{0} q_{z}\right) \\
& \times f\left[E_{N}+\left(E_{q_{z}}+\epsilon_{N^{\prime} N m}^{\mp}\right)^{2} / 4 E_{q_{z}}\right] .
\end{aligned}
$$

Here, $\quad \epsilon_{N^{\prime} N m}^{\mp}=\left(N^{\prime}-N\right) \hbar \omega_{c}-m \hbar \omega \mp \hbar \omega_{\mathrm{LO}}, \quad X_{N^{\prime} N}(y)$ $=\int_{0}^{\infty} d x C_{N^{\prime} N}(x) /(x+y)$, and $E_{q_{x}}=\hbar^{2} q_{z}^{2} / 2 m^{*}$.

For the case of a weak probing field $E_{z}$, the electronenergy-loss rate induced by $E_{z}$ is very small and $P \sim E_{z}^{2}$ $\ll 1$. Thus,

$$
P_{\mathrm{op}}=\sum_{m} m \hbar \omega \lambda_{m}^{\mp} \simeq P_{\mathrm{LO}}=\sum_{m}(\mp) \hbar \omega_{\mathrm{LO}} \lambda_{m}^{\mp},
$$

where $P_{\mathrm{op}}$ and $P_{\mathrm{LO}}$ are the EELR's induced, respectively, by optical and phonon scattering processes. Equation (11) reflects the fact that the energy an electron gains from the radiation field via optical absorption and emission is balanced by the energy an electron loses via emission and absorption of phonons. Furthermore, the energy gain in an optical process is the summation over the processes caused by phonon emission and absorption, whereas the energy loss in a phonon process is the energy difference between phonon emission and absorption.

For an electron gas system with relatively low electron density and at relatively high temperature or high excitation level, the Maxwellian with an electron temperature $T_{e}$, i.e., $f(x)=c e^{-\beta x}$, is the most popularly used statistical electronenergy-distribution function, where $\beta=1 / k_{B} T_{e}$ and $c$ is a normalization factor determined by the condition of electron number conservation via $c=2 \pi N_{e} l^{2} \sqrt{\pi \beta} /\left(A \sqrt{2 m^{*} / \hbar^{2}}\right)$ with $A=\Sigma_{N} e^{-\beta E_{N}}$. Using this distribution function, the momentum- and energy-relaxation times can be calculated simply through 


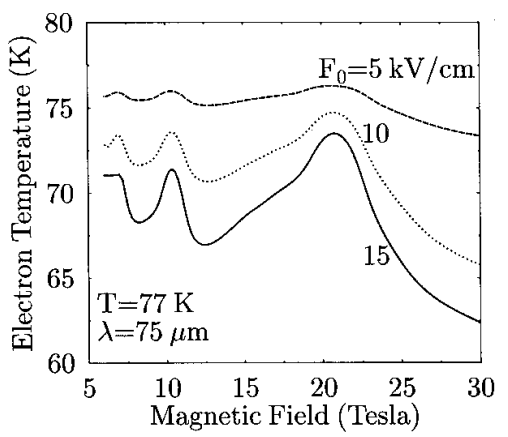

FIG. 1. Electron temperature as a function of magnetic field for different radiation intensities $F_{0}$ at a fixed lattice temperature $T$ and a fixed radiation wavelength $\lambda=75 \mu \mathrm{m}$ (or $f=4 \mathrm{THz}$ ).

$$
\begin{aligned}
\frac{1}{\tau}= & \omega_{\mathrm{LO}} \frac{2 \alpha \beta}{A} \sqrt{\beta \hbar \omega_{\mathrm{LO}} / \pi}\left[N_{0}+\frac{1}{2} \mp \frac{1}{2}\right] \sum_{m=-\infty}^{\infty} \sum_{N^{\prime}, N} e^{-\beta E_{N}} \\
& \times \int_{0}^{\infty} \frac{d q_{z}}{q_{z}} X_{N^{\prime} N}\left(l^{2} q_{z}^{2} / 2\right) J_{m}^{2}\left(r_{0} q_{z}\right) \\
& \times\left(E_{q_{z}}+\epsilon_{N^{\prime} N m}^{\mp}\right) e^{-\beta\left(E_{q_{z}}+\epsilon_{N^{\prime} N m}^{\mp}\right)^{2} / 4 E_{q_{z}}}
\end{aligned}
$$

and

$$
\begin{aligned}
\lambda_{m}^{\mp}= & \omega_{\mathrm{LO}} \frac{\alpha}{A} \sqrt{\beta \hbar \omega_{\mathrm{LO}} / \pi}\left[N_{0}+\frac{1}{2} \mp \frac{1}{2}\right] \sum_{N^{\prime}, N} e^{-\beta E_{N}} \\
& \times \int_{0}^{\infty} \frac{d q_{z}}{q_{z}} X_{N^{\prime} N}\left(l^{2} q_{z}^{2} / 2\right) J_{m}^{2}\left(r_{0} q_{z}\right) e^{-\beta\left(E_{q_{z}}+\epsilon_{N^{\prime} N m}^{\mp}\right)^{2} / 4 E_{q_{z}} .}
\end{aligned}
$$

\section{NUMERICAL RESULTS AND DISCUSSION}

In this paper, the numerical calculations are carried out for bulk GaAs. The material parameters for GaAs are taken as (i) effective electron mass $m^{*}=0.0665 m_{e}$ with $m_{e}$ being the rest electron mass; (ii) electron-LO-phonon coupling constant $\alpha=0.068$; and (iii) LO-phonon energy $\hbar \omega_{\mathrm{LO}}$ $=36.6 \mathrm{meV}$. The optical channels for $m=0, \pm 1, \pm 2, \ldots$, \pm 25 and the Landau levels for $N=0,1,2, \ldots, 5$ are included within the numerical calculations. Inclusion of more optical processes and more LL's affects only the results for lowfrequency and very high-intensity radiation and for low magnetic fields. Moreover, for the calculation of the resistivity, we take a typical electron density in $n$-type-doped GaAs: $N_{e}=10^{23} \mathrm{~m}^{-3}$

By solving the energy-balance equation, Eq. (11), we can obtain the electron temperature $T_{e}$ and then calculate the power induced by optical $P_{\text {op }}$ and phonon $P_{\text {LO }}$ scattering processes. Introducing $T_{e}$ into the momentum-balance equation, we can determine the momentum-relaxation time and resistivity $\rho_{z z}$.

The dependence of electron temperature on magnetic field is shown in Fig. 1 at a fixed radiation frequency and a fixed lattice temperature for different radiation intensities. ${ }^{9}$ We see that in the presence of intense $\mathrm{THz}$ radiation the MPR effect

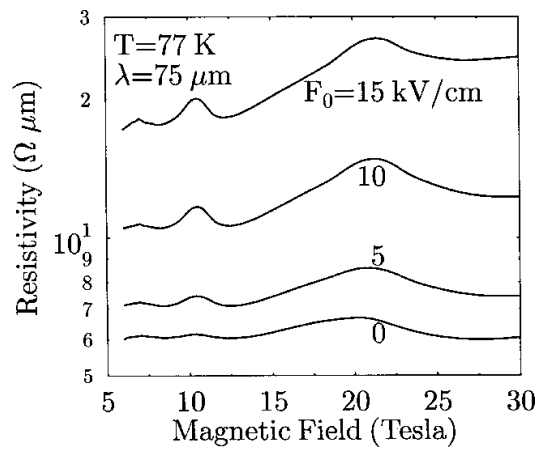

FIG. 2. Resistivity $\rho_{z z}$ versus magnetic field at a fixed radiation frequency for different radiation intensities. $F_{0}=0$ is the case in the absence of the radiation field.

can be observed by measuring the electron temperature. With increasing radiation intensity, a more marked MPR effect appears. It is interesting to note that at a wavelength $\lambda$ $=75 \mu \mathrm{m}$ (or a frequency $f=4 \mathrm{THz}$ ) the presence of the $\mathrm{THz}$ radiation results in a cooling of the electron gas, i.e., $T_{e}$ $<T$. In this case, the process for an electron to lose energy via LO-phonon emission is much more efficient than that to gain energy via photon absorption, due to the large energy transfer caused by LO-phonon scattering. As a result, the requirement $P_{\mathrm{op}} \simeq P_{\mathrm{LO}}$ leads to lowering $T_{e}$ so that LOphonon absorption becomes stronger. This is the main reason why a stronger MPR occurs when intense $\mathrm{THz}$ radiation is present. The cooling of electrons through a similar mechanism was already seen in nonlinear electron transport in strong dc fields ${ }^{10}$ and in the presence of a weak magnetic field. ${ }^{11}$

The resistivity $\rho_{z z}$ as a function of magnetic field is shown in Fig. 2 at a fixed radiation frequency for different radiation intensities. $F_{0}=0$ is the case where the radiation field is absent. In the presence of $\mathrm{THz}$ radiation, electrons in the system can gain energy from the radiation field via absorption of photons (see Fig. 3) and lose energy by emission of LO phonons. This two-step process opens up new channels for electronic transitions and, therefore, a larger resistivity can be found when $\mathrm{THz}$ radiation is present. With increasing radiation intensity, more channels for optical emission and

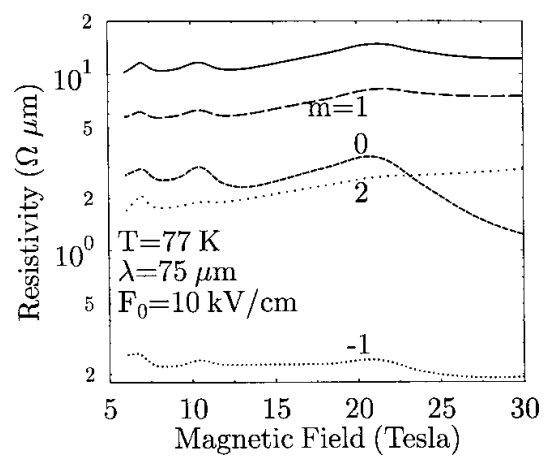

FIG. 3. Contribution from different optical channels to resistivity $\rho_{z z}$ as a function of magnetic field at a fixed radiation field. $m>0(m<0)$ corresponds to a contribution from $m$-photon absorption (emission) and the solid curve is the total resistivity. 


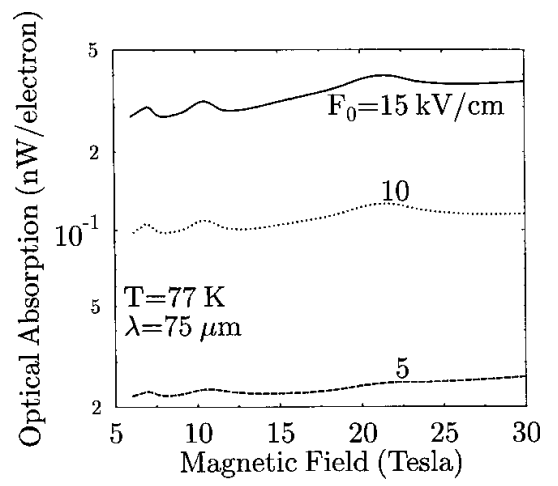

FIG. 4. Power of optical absorption per electron as a function of magnetic field at a fixed radiation frequency for different radiation intensities.

absorption open up and the resistivity increases. Similar to what we have seen in Fig. 1, a stronger MRP effect can be observed in $\rho_{z z}$ at a higher laser excitation level. When an electron gas is subjected to intense $\mathrm{THz}$ fields and quantizing magnetic fields in the Voigt geometry, the emission and absorption of photons are indirect optical processes that are mediated by electron-phonon scattering. As a result, the resistivity of the system is the summation over all possible optical processes. The contribution from different optical channels to resistivity is plotted in Fig. 3 as a function of magnetic field at a fixed radiation field. We notice that optical absorption $(m>0)$ and elastic optical $(m=0)$ processes contribute mainly to the resistivity and that the MPR effect can be observed for different optical processes.

The power of optical absorption per electron is plotted in Fig. 4 as a function of magnetic field at a fixed radiation frequency for different radiation intensities, and the contribution from different optical channels to optical absorption at a fixed radiation field is shown in Fig. 5. From these results, we find that in the presence of intense laser radiation (i) the MPR effect can be measured not only by transport experiments but also by optical measurements, because the optical absorption coefficient is proportional to the power of optical

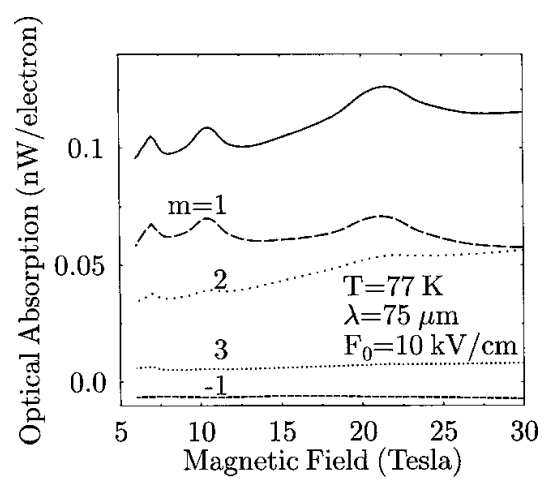

FIG. 5. Contribution from different optical processes to power of optical absorption as a function of magnetic field at a fixed radiation field. $m>0(m<0)$ corresponds to a contribution from $m$-photon absorption (emission) and the solid curve is the total power of optical absorption per electron.

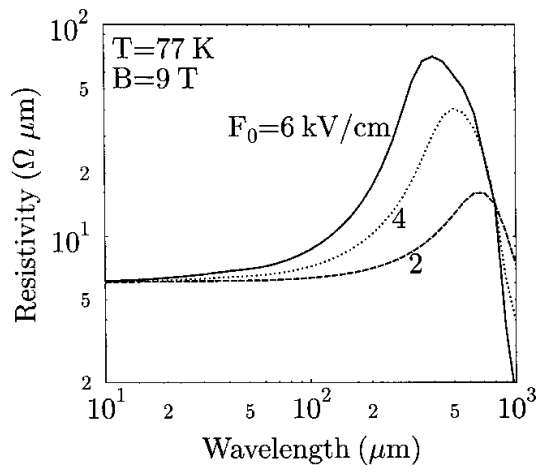

FIG. 6. Resistivity $\rho_{z z}$ as a function of radiation wavelength for different radiation intensities at a fixed lattice temperature and a fixed magnetic field.

absorption per electron; ${ }^{12}$ (ii) with increasing radiation intensity, a stronger MPR effect can be found in the optical absorption coefficient; and (iii) the MPR effect can also be seen in the optical absorption coefficient for different optical processes (see Fig. 5).

The dependence of resistivity on radiation frequency is shown in Fig. 6 at a fixed magnetic field for different radiation intensities. We note that there is an absorption peak at about $\lambda \sim 300 \mu \mathrm{m}$ (or $f \sim 1 \mathrm{THz}$ ). With increasing radiation intensity, a stronger absorption can be observed and the absorption peak shifts to the higher-frequency regime. In 1995, Asmar et al. measured the frequency dependence of the $\mathrm{THz}$ absorption in different 2DEG samples including GaAs-based heterojunctions and single quantum wells in the absence of a magnetic field. ${ }^{13}$ At $B=0$ they also found a peak of resonant absorption at about $f \sim 0.5 \mathrm{THz}$ at moderate electron temperatures $\left(T_{e} \sim 80 \mathrm{~K}\right)$ or radiation excitation levels. As can be seen from Fig. 7 , at about $\lambda \sim 300 \mu \mathrm{m}$ (or $f \sim 1 \mathrm{THz}$ ) the strongest magnetophoton-phonon scattering occurs via channels for optical absorption including multiphoton absorption processes. When this happens, the electrons in the system are heated and can gain energy from the radiation field and lose it by emission of LO phonons. This process opens up new channels for electronic transitions via resonant

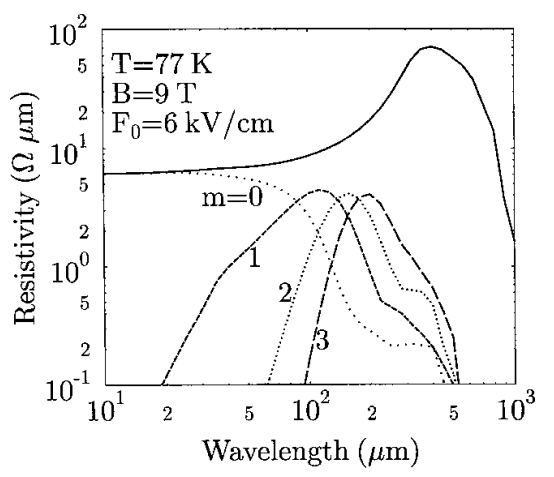

FIG. 7. Contribution from different optical channels to resistivity as a function of radiation wavelength at a fixed radiation intensity and a fixed magnetic field. $m>0$ corresponds to a contribution from $m$-photon absorption and the solid curve is the total resistivity. 


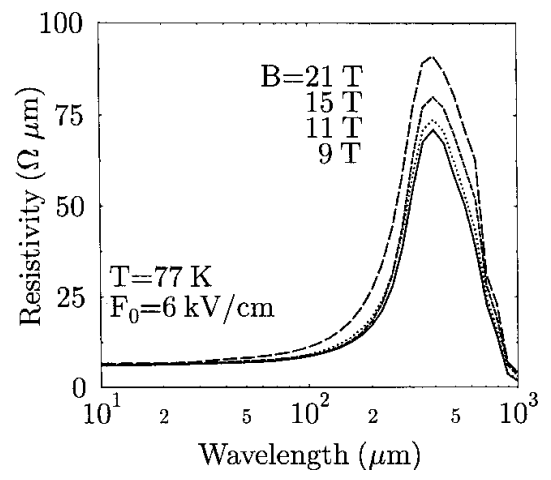

FIG. 8. Resistivity $\rho_{z z}$ versus radiation wavelength at a fixed radiation intensity for different magnetic fields.

magnetophoton-phonon scattering and, as a result, the resistivity increases and an optical absorption peak can be observed in the resistivity. From these theoretical results, we conclude that the resonant-absorption peak observed at about $f \sim 1 \mathrm{THz}$ in the presence of magnetic fields is electrically analogous to those observed experimentally at about $f$ $\sim 0.5 \mathrm{THz}$ at $B=0$. Moreover, the results obtained from further calculations (see Fig. 8) show that at a fixed radiation intensity the peak of the resonant absorption does not shift with varying magnetic field.

\section{SUMMARY}

In this paper, we have developed a simple theoretical approach to dealing with electron interactions with an intense laser field in an electron-photon-phonon system in the presence of a quantizing magnetic field in the Voigt geometry. The electronic transition rate induced by magnetophotonphonon interaction has been obtained for an ideal 3DEG system. Introducing this electronic transition rate into the Boltzmann equation, we have derived the momentum- and energy-balance equations, which can be used to calculate the transport and optical coefficients for the present system. Furthermore, we have carried out the numerical calculations for electron temperature, resistivity, and power of the optical ab- sorption as functions of radiation and magnetic fields.

When an electron gas is subjected to quantizing magnetic fields and an intense $\mathrm{THz}$ laser field in the Voigt geometry, dramatic $\mathrm{THz}$ radiation phenomena can be observed in magnetotransport quantities such as the resistivity and electronenergy-loss rate. The physical reason behind this is that in the presence of intense $\mathrm{THz}$ radiation electrons can gain energy from the radiation field and lose it by emission of phonons. This opens up new channels for electronic transitions and, therefore, the excitation and relaxation of electrons are modified strongly by the radiation field. Thus, the magnetotransport coefficients depend strongly on the frequency and intensity of the $\mathrm{THz}$ field. We have found that the presence of intense $\mathrm{THz}$ radiation can result in an enhanced magnetophonon resonance effect in polar semiconductors such as GaAs. Furthermore, the resonant magnetophoton-phonon scattering can lead to an absorption peak observed at about $f \sim 1 \mathrm{THz}$ for GaAs within a wide magnetic field regime.

The present work is motivated by recent development and application of $\mathrm{THz}$ free-electron lasers as intense radiation sources in the investigation into optoelectronic properties of semiconductor systems. ${ }^{6,13-15}$ When a GaAs-based electron gas is subjected to a quantizing magnetic field and a laser field with a frequency $f \sim 1 \mathrm{THz}$ and an intensity $F_{0}$ $\sim 10 \mathrm{kV} / \mathrm{cm}$ (or output power $I \sim 100 \mathrm{~kW} / \mathrm{cm}^{2}$ ), ${ }^{6}$ conditions such as $\omega \sim \omega_{\mathrm{LO}} \sim \omega_{c}$ and $r_{0} q \sim 1$ can be satisfied. As a consequence, the photon-modified MPR effect can be observed and the effects caused by magnetophoton-phonon coupling can be examined. These radiation conditions have been realized by the current generation of THz FEL's. We therefore hope this work can provide a theoretical backup to experiments and experimental findings.

\section{ACKNOWLEDGMENTS}

W.X. acknowledges financial support from the Australian Research Council. This work was also supported by the Australian Academy of Science and Japan Society for the Promotion of Science. Discussions with P. M. Koenraad (TUE) and R. A. Lewis (UoW) are gratefully acknowledged.
*Electronic address: wxu@wumpus.its.uow.edu.au

${ }^{1} 1 \mathrm{THz}$ in frequency is $299.79 \mu \mathrm{m}$ in wavelength, $4.135 \mathrm{meV}$ in energy, and $47.99 \mathrm{~K}$ in temperature. For GaAs, $1 \mathrm{THz}$ is $2.376 \mathrm{~T}$ in magnetic field.

${ }^{2}$ For recent development of the FEL, see, e.g., Free Electron Lasers 1999, edited by J. Feldhaus and H. Weise (Elsevier Science, North-Holland, Amsterdam, 2000).

${ }^{3}$ W. Xu and C. Zhang, Phys. Rev. B 54, 4907 (1996); S. Y. Liu and X. L. Lei, ibid. 60, 10624 (1999).

${ }^{4}$ In the Voigt geometry, W. Xu, J. Phys.: Condens. Matter 9, L591 (1997).

${ }^{5}$ In the Faraday geometry, W. Xu and C. Zhang, Phys. Rev. B (to be published).

${ }^{6}$ P. M. Koenraad, R. A. Lewis, L. R. C. Waumans, C. J. G. M.
Langerak, W. Xu, and J. H. Wolter, Physica B 256-258, 268 (1998).

${ }^{7}$ For recent theoretical and experimental work, see, e.g., X. G. Wu, F. M. Peeters, Y. J. Wang, and B. D. McCombe, Phys. Rev. Lett. 84, 4934 (2000), and references about the CR effect in semiconductors therein.

${ }^{8}$ W. Xu, J. Phys.: Condens. Matter 10, 6105 (1998).

${ }^{9}$ The connection between the electric field strength of a laser field $\left(F_{0}\right)$ and the laser output power $(I)$ is $I=0.5 \sqrt{\epsilon / \mu}\left|F_{0}\right|^{2}$ $\simeq 1.32\left|F_{0}\right|^{2} \mathrm{~kW} / \mathrm{cm}^{2}$ where $F_{0}$ is in units of $\mathrm{kV} / \mathrm{cm}$.

${ }^{10}$ W. Xu, F. M. Peeters, and J. T. Devreese, Phys. Rev. B 43, 14134 (1991).

${ }^{11}$ W. Xu, F. M. Peeters, and J. T. Devreese, Phys. Rev. B 46, 7571 (1992). 
${ }^{12}$ X. L. Lei, J. Appl. Phys. 84, 1396 (1998).

${ }^{13}$ N. G. Asmar, A. G. Markelz, E. G. Gwinn, J. Cerne, M. S. Sherwin, K. L. Campman, P. F. Hopkins, and A. C. Gossard, Phys. Rev. B 51, 18041 (1995).

${ }^{14}$ K. B. Nordstrom, K. Johnsen, S. J. Allen, A. P. Jauho, B. Birnir, J.
Kono, T. Noda, H. Akiyama, and H. Sakaki, Phys. Rev. Lett. 81, 457 (1998).

${ }^{15}$ B. N. Murdin, W. Heiss, C. J. G. M. Langerak, S.-C. Lee, I. Galbraith, G. Strasser, E. Gornik, M. Helm, and C. R. Pidgeon, Phys. Rev. B 55, 5171 (1997). 